\title{
Recherches féministes
}

\section{La conciliation travail-famille : l'organisation policière canadienne en transformation? \\ Balancing Work and Family : Transforming Canadian Police \\ Force Management? \\ Conciliación trabajo y familia : ¿la organización policial canadiense en transformación?}

\section{Michèle Diotte}

Volume 29, numéro 2, 2016

URI : https://id.erudit.org/iderudit/1038728ar

DOI : https://doi.org/10.7202/1038728ar

Aller au sommaire du numéro

Éditeur(s)

Revue Recherches féministes

ISSN

0838-4479 (imprimé)

1705-9240 (numérique)

Découvrir la revue

Citer cet article

Diotte, M. (2016). La conciliation travail-famille : l'organisation policière canadienne en transformation? Recherches féministes, 29(2), 215-230.

https://doi.org/10.7202/1038728ar
Résumé de l'article

La féminisation des corps policiers canadiens a transformé certains éléments de la culture organisationnelle et oblige à repenser la conciliation travail-famille dans ce milieu. Les rapports sur les questions d'égalité homme-femme et de diversité au sein de la police se sont multipliés au cours des dernières années et laissent entrevoir la transformation d'un modèle traditionnellement masculin en un modèle plus inclusif. Le regard féministe invite à remettre en question ce propos, car entre discours et « pratique » il y a souvent un écart. L'auteure met donc en lumière l'état des mesures qui existent actuellement dans les grands corps policiers du pays relativement à la conciliation travail-famille et s'interroge sur la matrice dans laquelle s'articule la réflexion sur ce sujet. 


\section{La conciliation travail-famille: l'organisation policière canadienne en transformation?}

\section{MICHÈLE DIOTTE}

L'organisation policière canadienne se définit particulièrement par une gestion paramilitaire ${ }^{1}$ et en fonction de sa particularité opérationnelle (Agocs, Langan et Sanders 2015; Beauchesne 2007; Duxbury et Higgins 2012) ${ }^{2}$. Bien que la présence féminine au sein des corps policiers canadiens se soit accrue significativement au cours des dernières décennies ${ }^{3}$, Agocs, Langan et Sanders (2015) soulignent la persistance de la culture hypermasculine de la police. Les quelques recherches canadiennes portant précisément sur les policières, ainsi que la littérature plus générale à propos de la police, mettent en lumière un certain nombre de défis propres aux femmes dans la police (Agocs, Langan et Sanders 2015; Beauchesne 2007; Diotte 2001; GRC 2012). Le clivage entre les sphères familiale et professionnelle, préconisé par la culture policière, rend la conciliation des rôles de parent et de policier ou de policière particulièrement difficile (Agocs, Langan et Sanders 2015; Diotte 2001; Duxbury et Higgins 2012). À ce sujet, les membres des corps policiers revendiquent une plus grande prise en considération de leur situation familiale par l'organisation policière (Diotte 2001; Tremblay, Génin et Di Loreto 2011).

Dans le but de poursuivre la réflexion à propos de la conciliation travailfamille au sein de l'organisation policière canadienne, la présente recherche pose deux principales questions : comment s'articule la conciliation travail-famille à travers les politiques des grands corps policiers canadiens? À travers quelle grille d'analyse se réfléchit la conciliation travail-famille au sein de l'organisation policière? C'est par l'analyse documentaire, à la fois des conventions collectives en vigueur et des différents rapports récemment produits par les grands corps policiers canadiens, que nous tenterons de répondre à ces questions.

Ainsi, notre article se veut un espace pour réfléchir, dans une perspective féministe, sur le discours actuel entourant la conciliation travail-famille au sein de l'organisation policière et pour, dans une certaine mesure, prendre le pouls des

1 Les règles rigides, l'importance de la hiérarchie et la culture organisationnelle masculine peuvent être associées à cette gestion paramilitaire (Agocs, Langan et Sanders 2015; Beauchesne 2007).

2 Le travail de première ligne et les horaires de travail du type rotatif caractérisent, notamment, le travail policier (Beauchesne 2007).

3 Selon Statistique Canada (2015: 15), « [1] a proportion de policières au pays est à la hausse ces dernières décennies. Par exemple, en 1994, 9,1\% des policières étaient des femmes, comparativement à $16,5 \%$ en 2004 et à $20,6 \%$ en 2014 ».

Recherches féministes, vol. 29, n ${ }^{\circ}$ 2, $2016: 215-230$ 
DIOTTE | 216

pratiques mises en place par les grands corps policiers du pays à ce sujet. L'exploration de la grille d'analyse à travers laquelle s'opère le questionnement en matière de conciliation travail-famille et certains constats qui émergent de la littérature au sujet de cette conciliation permettront de mieux saisir la portée des mesures en vigueur dans les grands services de police au Canada. Nous verrons, d'une part, que les pratiques sont loin d'être uniformes d'un océan à l'autre et que, d'autre part, il y a lieu de remettre en question la grille d'analyse dans laquelle s'articulent les mesures de conciliation travail-famille.

\section{Une grille d'analyse où se réfléchit la conciliation travail-famille}

Au cours des dernières années, plusieurs corps policiers canadiens ont amorcé une réflexion sur la question de l'égalité homme-femme ou à propos de la diversité (GRC 2012; Gouvernement du Manitoba 2013; Royal Newfoundland Constabulary 2011; Ontario Provincial Police 2011; Service de police de la Ville de Montréal $2006)^{4}$. La conciliation travail-famille revient dans plusieurs rapports comme piste de solution à certains défis organisationnels concernant les effectifs policiers (GRC 2012 et 2013; Ontario Provincial Police 2011; Royal Newfoundland Constabulary 2011). Dans un contexte politique où l'inclusion et la diversité sont au programme et que plusieurs organisations policières possèdent des objectifs à atteindre quant à la proportion de femmes au sein de leur effectif, il n'est pas étonnant de voir émerger un questionnement à propos des barrières au recrutement et à la rétention des femmes dans la police. Comme le souligne le Rapport sur l'égalité des sexes et le respect (2012) produit par la Gendarmerie royale du Canada (GRC), la famille, la mobilité et la conciliation travail-famille sont d'importants éléments qui influent sur le recrutement, la promotion et la rétention des femmes dans la profession. Ainsi, la question de la conciliation travail-famille à l'intérieur de l'organe policier canadien s'articule particulièrement en termes de stratégie de recrutement et de maintien des effectifs féminins.

Avant d'aller plus loin, nous jugeons important de définir le concept de conciliation travail-famille, tel que nous l'avons utilisé dans notre recherche. La thématique travail-famille peut se définir comme une incompatibilité ou un conflit entre les rôles et les demandes liés à la sphère familiale et ceux qui se rattachent à la sphère professionnelle (Greenhaus et Beutell 1985; St-Amour et autres 2005; Tremblay, Di Loreto et Génin 2009; Tremblay et Génin 2011; Tremblay, Génin et Di Loreto 2011). Selon Greenhaus et Beutell (1985), ce conflit travail-famille peut prendre trois formes : 1) le conflit de temps; 2) la tension entre les rôles; et 3) le conflit de comportement. L'Association canadienne pour la santé mentale, Division de

$4 \quad$ Ici, la diversité fait référence principalement à la diversité culturelle. Elle s'articule dans une logique de représentativité de la population servie. Le rapport de l'Ontario Provincial Police (2011) en est un bon exemple. 
LA CONCILIATION TRAVAIL-FAMILLE : L'ORGANISATION POLICIÈRE | 217

l'Ontario (2015), ajoute la sphère communautaire à celles de la famille et du travail. La conciliation travail-famille peut également se définir comme « a state of well-being that a person can reach or can set as a goal in order to allow them to manage effectively multiple responsibilities at work, at home and in their community » (Association canadienne pour la santé mentale, Division de l'Ontario 2015). Plus particulièrement, les mesures de conciliation travail-famille tendent à réduire le conflit existant entre ces sphères et à faciliter l'organisation du temps et des responsabilités des salariés (Tremblay, Di Loreto et Génin 2009). En rapport avec cette définition, quels sont les aspects organisationnels qui devraient être revus, remis en question et transformés pour réduire le conflit travail-famille au sein de l'organisation policière?

\section{La manière de favoriser la conciliation travail-famille}

Les recherches sur les policières et sur la conciliation travail-famille dans l'organisation policière, ainsi que la littérature plus générale sur le conflit travailfamille, mettent en lumière un certain nombre d'avenues pour faciliter cette conciliation dans l'organisation policière. Le type d'horaire qui existe dans cette profession est régulièrement désigné par la littérature comme un défi intimement lié à l'articulation de la vie professionnelle et de la vie personnelle (Duxbury et Higgins 2012; GRC 2013; Statistique Canada 2008; Tremblay, Génin et Di Loreto 2011). Les horaires atypiques, qui sont basés sur trois relèves et demandent aux policières et aux policiers de travailler en alternance selon des horaires de jour, de soir et de nuit, semblent constituer une source de stress, particulièrement pour celles qui sont mères de famille (Agocs, Langan et Sanders 2015; Diotte 2001; Kurtz 2012). Dans ce type d'horaire, la question de la garde des enfants est un enjeu pouvant devenir un véritable casse-tête pour les couples policiers (Diotte 2001) . $^{5}$ Dans la plupart des cas, les couples policiers dépendent de l'ouverture du supérieur, cette personne décidant de tenir compte, ou non, de leur situation dans l'élaboration des horaires (Schulze 2011; Diotte 2001). Toutefois, si les horaires atypiques peuvent être source de stress, ils sont aussi considérés, dans certains contextes, comme un élément facilitant la conciliation travail-famille. À ce sujet, Fusulier, Sanchez et Ballatore (2013) de même que Tremblay, Génin et Di Loreto (2011) soulignent que c'est le cas lorsqu'il y a possibilité d'arrangements informels et présence d'un soutien organisationnel, tant de la part de la direction que des collègues. Le type d'horaire qui règne au sein de l'organisation policière pourrait donc être à la fois allié et ennemi de la conciliation travail-famille. La manière dont ces horaires atypiques sont vécus dépend possiblement du degré de souplesse d'horaire présent dans l'organisation, de la légitimité donnée par le service de police quant aux possibles arrangements et du

5 Le concept de couple policier fait référence à deux personnes occupant la même fonction et entretenant ensemble une relation amoureuse. Selon Diotte (2001 : 18), environ la moitié des policières canadiennes sont en situation de couple avec un policier ou une policière. 
soutien organisationnel concernant la question de la garde des enfants (Diotte 2001; Duxbury et Higgins 2012; Fusulier, Sanchez et Ballatore 2012; Tremblay, Génin et Di Loreto 2011).

L'accessibilité au travail à temps partiel et le partage de poste sont d'autres pistes qui ressortent des réflexions à propos de la conciliation travail-famille. La littérature met en lumière certains enjeux liés à ces avenues. D'une part, il importe que le temps partiel ou le partage de poste soit de qualité, c'est-à-dire qu'il offre les mêmes avantages sociaux, conditions de travail, accès aux formations et aux promotions que le temps plein, en fonction du prorata des heures travaillées (Charlesworth, Keen et Whittenbury 2009). Sans ces conditions, ces options resteront sous-utilisées ou encore risquent de produire des emplois à deux vitesses dont les femmes, généralement, paient le prix. D'autre part, l'efficacité de ces mesures exige qu'elles soient légitimées et promues au sein même de l'organisation policière (GRC 2013). En effet, pour être perçus comme des avenues à considérer, le travail à temps partiel et le partage de poste doivent être vus comme des pratiques cohérentes avec la culture professionnelle. Il importe donc pour l'organisation policière de revoir certaines croyances qui subsistent, telles que l'idée d'une scission entre les sphères professionnelle et personnelle, la priorisation du travail sur la famille et le rendement se mesurant sous forme d'heures travaillées (Agocs, Langan et Sanders 2015; Charlesworth, Keen et Whittenbury 2009; Duxbury et Higgins 2012; Fusulier, Sanchez et Ballatore 2013).

La question des congés est également abordée dans la littérature à propos de l'organisation policière et de la conciliation travail-famille (Fusulier, Sanchez et Ballatore 2013; GRC 2013; Montgomery 2012; Tremblay et Génin 2011). Nous distinguons ici deux types de congés, soit le congé prolongé, tels les congés de maternité/paternité/parental et les congés autofinancés (congé sans solde ou différé), ainsi que le congé ponctuel, notamment le congé pour obligation familiale. Au Canada, les congés de maternité, de paternité et parental ${ }^{6}$ sont gérés par le Régime d'Assurance-emploi au fédéral ou par le Régime québécois d'assurance parentale ${ }^{7}$. Ces politiques canadiennes et québécoises en matière de congés à la suite de la naissance ou de l'adoption d'un ou d'une enfant offrent des conditions précises en fait de durée et de rémunération, mais celles-ci peuvent être bonifiées par l'organisation policière. Comme ces congés s'articulent autour des droits parentaux selon les normes du travail qui existent dans chaque province, leur accès ne constitue pas un enjeu pour les membres des corps policiers. En ce qui concerne le congé autofinancé ou la «pause carrière », ces pratiques semblent peu documentées dans la littérature sur

6 Sont ici visés les différents régimes d'assurance parentale canadien (www.educaloi.qc.ca/en/capsules/maternity-paternity-and-parental-leave) et québécois (www.rqap.gouv.qc.ca/travailleur_salarie/choix.asp).

7 L'article de Tremblay et Génin (2011) ainsi que l'ouvrage de Tremblay et Lazzari Dodeler (2000) discutent plus longuement de la spécificité du congé parental au Québec. 
LA CONCILIATION TRAVAIL-FAMILLE : L'ORGANISATION POLICIÈRE $\mid 219$

l'organisation policière canadienne ${ }^{8}$, bien que ce type de congé soit indiqué dans quelques rapports produits par des organisations policières canadiennes comme une des pistes à explorer pour favoriser la conciliation travail-famille (GRC 2013; Montgomery 2012). Il en ressort que les congés prolongés peuvent avoir un effet négatif, tant sur l'employé ou l'employée qui s'en prévaut que sur ses collègues et l'organisation policière, si cette dernière ne prend pas soin de remplacer la personne qui quitte temporairement son poste (Busson 1997; GRC 2013).

La question des congés ponctuels, quant à elle, se présente différemment. D'une part, la littérature précise que la gestion du temps est une source de stress pour les policières et les policiers qui ont une famille (Duxbury et Higgins 2012; Kurtz 2012). D'autre part, dans une étude exploratoire menée auprès de policières québécoises, plusieurs réclamaient des congés familiaux afin de ne pas avoir à utiliser leurs propres congés de maladie pour des situations en rapport avec leurs obligations parentales (Diotte 2001). Ici, l'enjeu est plus que la simple souplesse d'horaire abordée précédemment : il concerne la rémunération et la reconnaissance de la dimension familiale du personnel par l'octroi d'une banque de journées rémunérées précisément réservée à la conciliation travail-famille.

De façon générale, la littérature traitant de l'organisation policière et des questions liées à la famille soutient que le conflit entre le travail et la famille est une problématique plus accentuée chez les femmes (Agocs, Langan et Sanders 2015; Tremblay et Lazzari Dodeler 2000). Bien qu'une très grande proportion de policiers soient également parents (Duxbury et Higgins 2012), les policières semblent, encore de nos jours, être celles qui portent plus souvent la responsabilité familiale et qui assument une grande partie du travail domestique (Agocs, Langan et Sanders 2015; Tremblay et Lazzari Dodeler 2000). Également, la situation familiale dans laquelle se trouvent beaucoup plus de policiers que de policières, où la prise en charge des enfants est principalement assumée par la conjointe ou le conjoint, peut contribuer à nourrir l'idée que les enjeux liés à la conciliation travail-famille concerne davantage les femmes (Duxbury et Higgins 2012; Schulze 2011). En d'autres mots, la reconnaissance et les accommodements en rapport avec la famille paraissent jouer différemment selon le sexe sur la perception de l'engagement au sein de la profession (Charlesworth, Keen et Whittenbury 2009).

\section{La conciliation travail-famille à travers les politiques organisationnelles}

Les rapports de plusieurs organisations policières canadiennes indiquent qu'une réflexion sur la conciliation travail-famille est entreprise et qu'elle prend racine essentiellement dans un discours portant sur l'égalité homme-femme et la

8 Selon une recherche belge, la «pause carrière » est jugé par les policières et les policiers comme une pratique ne faisant pas partie de la culture professionnelle (Fusulier, Sanchez et Ballatore 2013). 
DIOTTE | 220

diversité (GRC 2012; Gouvernement du Manitoba 2013; Royal Newfoundland Constabulary 2011; Ontario Provincial Police 2011; Service de police de la Ville de Montréal 2006). La littérature américaine, européenne, australienne et canadienne met en lumière un certain nombre d'avenues favorisant la conciliation travail-famille au sein des forces policières. Avenues également mises en avant dans des rapports produits par la Gendarmerie royale du Canada (GRC 2012 et 2013). Cependant, comment se traduisent ces pistes de conciliation travail-famille dans les mesures mises en place par les corps policiers canadiens? Entre discours et pratiques, il y a souvent un décalage. L'analyse des conventions collectives ${ }^{9}$ en vigueur dans les grands corps policiers canadiens permet un début d'exploration de la façon dont s'imbriquent plus concrètement les éléments du discours en matière de conciliation travail-famille.

\section{La démarche méthodologique}

Dans l'objectif empirique de mettre en évidence les mesures organisationnelles favorisant la conciliation travail-famille en vigueur au sein des organisations policières du pays, les conventions collectives sont un matériau d'étude accessible et fiable, puisqu'il s'agit de documents officiels. Nous entendons par « convention collective » l'accord conclu entre l'organisation patronale et la partie syndicale (qui représente les personnes salariées) en vue de s'entendre sur les conditions d'emploi et sur les garanties sociales qui s'y rattachent (Hébert et autres 2007). Il importe cependant de nommer un certain nombre de limites liées à ce type de données. D'une part, les conventions collectives en vigueur ne reflètent pas nécessairement l'ensemble des pratiques organisationnelles : elles sont généralement décalées des façons de faire qui s'opèrent sur le terrain. D'autre part, elles portent sur une période de temps déterminée, variant de une à cinq années (leur durée peut s'étirer encore plus loin dans le temps lorsque les conventions collectives sont en négociation), et elles offrent un portrait des mesures historiquement situées dans une courte période de temps. Pour les besoins de l'analyse, les conventions des corps policiers ayant des effectifs de 1500 personnes et plus en 2013 (Statistique Canada 2014) ont été retenues, les probabilités étant plus grandes de trouver, dans ces corps policiers, un effectif féminin plus nombreux que ceux des services de police de moindre taille. À la lumière de ce critère, le corpus de conventions collectives a été limité aux services policiers de grandes villes canadiennes et d'organisations policières provinciales (tableau ci-dessous). Quoique la GRC ait entamé une réflexion sur la question de la conciliation travail-famille, ses mesures ne font pas partie de

9 Il va sans dire que les conventions collectives ne peuvent offrir à elles seules un portrait de la conciliation travail-famille. Au plus, elles donnent des pistes pour une exploration future par l'entremise, notamment, d'entretiens semi-dirigés avec des personnes clés au sein de l'organisation policière. Notre choix quant à l'analyse des conventions collectives s'explique essentiellement par des contraintes de temps et par l'accessibilité à l'information. 
LA CONCILIATION TRAVAIL-FAMILLE : L'ORGANISATION POLICIÈRE | 221

notre analyse au même titre que les autres organisations policières, puisque les membres et les réservistes de la GRC n'ont pas encore le droit de prendre part aux négociations collectives. Le Gouvernement du Canada s'est toutefois engagé, en janvier 2015, à proposer un projet de loi à ce sujet ${ }^{10}$.

\section{Services policiers canadiens étudiés}

\begin{tabular}{|l|l|l|l|}
\hline Corps policier & $\begin{array}{l}\text { Type } \\
\text { d'organisation }\end{array}$ & $\begin{array}{l}\text { Effectif } \\
\text { total }\end{array}$ & Convention collective \\
\hline Winnipeg & Municipal & 1521 & $2012-2016$ \\
\hline Montréal & Municipal & 7194 & $2011-2014$ \\
\hline Toronto & Municipal & 9967 & $2011-2014$ \\
\hline Calgary & Municipal & 2130 & $2011-2014$ \\
\hline Edmonton & Municipal & 1985 & $\begin{array}{l}\text { De juillet à } \\
\text { décembre 2013 }\end{array}$ \\
\hline Vancouver & Municipal & 3645 & $2012-2015$ \\
\hline $\begin{array}{l}\text { Police provinciale de } \\
\text { l'Ontario (PPO) }\end{array}$ & Provincial & 6069 & $2009-2011$ \\
\hline $\begin{array}{l}\text { Sûreté du Québec } \\
\text { (SQ) }\end{array}$ & Provincial & 5762 & $2010-2015$ \\
\hline Source: Statique Cand & & \\
\hline
\end{tabular}

Source : Statistique Canada (2014), Police provinciale de l'Ontario (2015) et Sûreté du Québec (2014).

Pour faire ressortir les mesures qui favorisent la conciliation travail-famille en vigueur au sein des grands corps policiers canadiens durant la période 2009-2016, nous avons effectué l'analyse documentaire des conventions collectives ratifiées par l'employeur et la partie syndicale selon une grille par thèmes. Ces derniers apparaissent dans la littérature comme autant d'avenues qui favorisent, potentiellement, la conciliation travail-famille dans l'organisation policière : l'horaire de travail, le temps partiel/partage de poste, le congé autofinancé et le congé familial.

Il apparaît dans l'analyse des conventions collectives en vigueur que la grande majorité des corps policiers étudiés (7 sur 8 ) offre plus d'un type d'horaire de travail, variant de huit à douze heures de travail par jour. Certaines organisations policières (4 sur 8 ) proposent la formule des heures compressées à leur personnel ou à certaines unités de travail. Cette formule consiste à augmenter le nombre d'heures travaillées dans une journée afin de réduire le nombre de journées travaillées durant la semaine ou un cycle de travail. Soulignons que le choix des horaires de travail est généralement à la discrétion des chefs d'équipe. Les conventions collectives indiquent donc que

10 Le communiqué de presse est accessible sur le Web : nouvelles.gc.ca/web/articlefr.do?mt hd=advSrch\&crtr.mnthndVl=12\&crtr.mnthStrtVl=1\&crtr.page=1\&crtr.dpt1D=6694\&nid $=1023859 \&$ crtr.yrndVl=2015\&crtr.yrStrtVl=2015\&crtr.dyStrtVl=1\&crtr.dyndVl=31\&_g $\mathrm{a}=1.6760049 .216619434 .1439226559$ (20 mai 2016). 
différents types d'horaire sont accessibles, sans pour autant donner de l'information sur la propension de la direction à autoriser les demandes de changements d'horaire provenant des policiers ou des policières ${ }^{11}$. La flexibilité d'horaire peut aussi se réfléchir en fonction de l'échange et du remplacement. À ce sujet, un peu plus de la moitié des corps policiers étudiés (5 sur 8 ) abordent cette question dans leur convention collective. La majorité ne fait cependant qu'effleurer le sujet, alors qu'une organisation encadre davantage la pratique. De façon générale, l'échange d'un quart de travail ou le remplacement doit être autorisé et ne doit pas engendrer de coût supplémentaire pour l'organisation ou de perte pour les personnes visées. Aborder la question de l'échange ou du remplacement dans le contexte d'une convention collective a pour effet d'officialiser la pratique.

Le travail à temps partiel et le travail à temps partagé (partage de poste) sont deux avenues proposées par la littérature dans l'optique de favoriser la conciliation travail-famille des policières et des policiers. L'analyse des conventions collectives montre que l'option du travail à temps partiel est très peu courante. En effet, ce type de travail est offert seulement dans deux organisations policières à l'étude. Toutefois, le travail à temps partagé semble beaucoup plus répandu, puisqu'il apparaît dans la moitié des corps policiers étudiés (4 sur 8). Les propositions de travail à temps partagé ont en commun certains points, notamment la durée d'application d'une année, l'obligation que la demande de travail à temps partagé soit présentée par deux personnes qui occupent la même position et l'application des conditions de travail du personnel à temps plein au prorata des heures travaillées. Fait intéressant, deux corps policiers ont clairement souligné que cette option a pour objet de favoriser la conciliation travail-famille et de maintenir en poste des personnes qui, sans cette solution, quitteraient possiblement l'organisation policière. Les deux mêmes services de police spécifient également dans leur convention collective l'importance de recourir à un employé ou à une employée temporaire afin de compenser la perte de prestation découlant des ententes de temps partagé.

En matière de congé de maternité/paternité/parental, les organisations policières respectent le plan en vigueur dans leur province. Les différences s'inscrivent plutôt dans l'octroi ou non de prestations supplémentaires de chômage aux policières et aux policiers à l'occasion du congé de maternité, de paternité (offert seulement au Québec) ou parental. Les corps policiers offrant une prestation qui vient s'ajouter au Régime d'assurance-emploi au fédéral ou à celui du Régime québécois d'assurance parentale permettent à leurs membres de recevoir de 75 à $100 \%$ de leur revenu durant ces différents congés. Si une majorité des organisations policières (5 sur 8 ) offrent des prestations supplémentaires de chômage, deux de celles-ci

11 Il serait intéressant d'explorer dans une recherche future les pratiques en matière d'échange d'horaire et de remplacement. Cela offrirait plus amples renseignements sur la façon dont les mesures sont appliquées et permettrait de faire ressortir les enjeux possibles pour les policières. 
LA CONCILIATION TRAVAIL-FAMILLE : L'ORGANISATION POLICIÈRE $\mid 223$

octroient des prestations uniquement aux policières en congé de maternité et n'étendent pas la compensation au congé parental. Soulignons que la moitié des organisations policières étudiées (4 sur 8) spécifient la possibilité d'obtenir un congé sans solde à la suite du congé de maternité/parental, dont la durée varie de 15 semaines à une année, selon le service policier. En matière de congé sans solde, sans égard à la naissance ou à l'adoption d'un ou d'une enfant, on l'aborde clairement dans quatre conventions collectives, alors que la politique relative au congé différé est proposée dans trois conventions collectives. Les deux types de congés (sans solde et différé) sont généralement d'une durée maximale d'une année et exigent d'avoir travaillé pour le service de police depuis un nombre d'années déterminé avant d'y être admissible.

Finalement, les congés alloués pour obligations familiales sont certainement l'avenue la moins développée dans les conventions collectives à l'étude. Ainsi, quelques organisations policières ( 3 sur 8 ) indiquent clairement une banque de congés pour raisons familiales. Toutefois, une seule des trois offre un congé payé, à raison de trois jours par année. Les deux autres permettent aux policières et aux policiers de prendre jusqu'à dix jours par année pour vaquer à leurs obligations familiales, mais ces journées sont sans traitement. Deux autres conventions collectives stipulent qu'un nombre déterminé de jours (ou d'heures) peuvent être puisés dans la banque personnelle de congés de maladie afin de répondre aux besoins de santé de la famille immédiate. Dans le cas des trois conventions collectives restantes, aucune mention n'est faite en matière de congé pour obligations familiales.

\section{Des enjeux pour les femmes...}

Malgré les mesures mises en place en matière d'heures de travail compressées et de partage de poste dans la moitié des organisations policières étudiées, peu d'entre elles expriment clairement leur préoccupation concernant la conciliation travailfamille. Les services de police des villes de Montréal et de Toronto sont les deux seules organisations qui inscrivent plus précisément certaines de leurs mesures dans une perspective d'équilibre entre la vie professionnelle et la vie familiale de leur personnel. À titre d'exemple, le Service de police de Toronto mentionne que « $[\mathrm{t}] \mathrm{he}$ Board has adopted job sharing and reduced hours programs to assist members who have difficulty working current work schedules because of personal problems such as child care or elder care » (Toronto Police Association 2011 : 83).

Or, l'existence de mesures favorisant la conciliation travail-famille n'est pas suffisante. Encore faut-il que l'organisation policière en fasse la promotion et que l'option soit valorisée par l'employeur et les collègues, sans quoi les policières et les policiers qui ont besoin de ces mesures n'y auront pas recours ou n'obtiendront pas partout le même appui (GRC 2012; Tremblay 2012; Tremblay, Di Loreto et Génin 2009; Tremblay et Génin 2011; Tremblay, Génin et Di Loreto 2011). En d'autres mots, les valeurs véhiculées par la culture organisationnelle, tout comme le soutien organisationnel concret, sont au centre du questionnement à propos de la conciliation 
DIOTTE $\mid 224$

travail-famille au sein de la profession policière. Comme le soulignent Duxbury et Higgins (2012), il importe de procéder à des changements culturels à l'intérieur des organisations, en reconnaissant que la famille doit parfois avoir priorité sur le travail.

Cependant, comment opérer ces transformations? Actuellement, la grille d'analyse à travers laquelle est pensée la conciliation travail-famille au sein de l'organisation policière est celle de l'égalité homme-femme et de la diversité. Selon cette perspective, les corps policiers associent les mesures qui favorisent la conciliation travail-famille aux besoins des policières, d'où la logique d'égalité des sexes. Or, il y a lieu de se questionner sur les enjeux découlant de la féminisation de la conciliation travail-famille dans l'organisation policière. N'y a-t-il pas là un risque de ressac pour les policières? Comme le fait remarquer Beauchesne (2007 : 185), « les organisations policières n'ont pas à faire porter aux femmes ${ }^{12}$ des changements structurels qui relèvent de leur responsabilité » et qui concernent autant les policiers que les policières (Duxbury et Higgins 2012; Tremblay et Lazzari Dodeler 2000; Tremblay, Génin et Di Loreto 2011). Or, comme l'indiquent la GRC (2012) ainsi que Tremblay, Génin et Di Loreto (2011), l'équilibre entre carrière et vie personnelle devient une préoccupation générationnelle, en ce sens où les jeunes générations accordent une plus grande importance au temps en dehors du travail (vacances, accumulation des congés, etc.) et à la flexibilité des horaires (heures de travail et cycles de travail). L'équilibre entre vie professionnelle et vie privée est central pour ces jeunes policières et policiers qui ont comme attente une structure organisationnelle flexible (Gauthier 2008). Dans le même sens, l'étude menée par Duxbury et Higgins en 2012 auprès de 4500 membres des corps policiers travaillant au sein de 25 organisations policières canadiennes conclut que le sexe n'est pas un prédicteur significatif dans le fait de vivre un plus haut niveau d'interférence entre le travail et la famille. La situation de conflit entre travail et famille serait ainsi associée davantage à l'âge qu'au sexe des personnes interrogées (Duxbury et Higgins 2012 : 103) :

This form of work life conflict is experienced by younger police officers with significant family demands (responsible for childcare), significant work demands (understaffing, multiple competing demands, work outside their mandate) and an inability to control either their family circumstances (family incomes are tight which would make it harder for the officer pay for childcare, especially when they are working shifts) or their work circumstances (low control over work, shift work, culture which discourages officers from seeking help).

L'association faite entre la conciliation travail-famille et les femmes dans le discours organisationnel d'égalité des sexes et de diversité n'est-elle pas à double

12 Rappelons que les femmes sont encore minoritaires dans l'organisation policière. Elles représentaient $20 \%$ de l'effectif policier en 2014 selon Statistique Canada (2015 : 15). 
tranchant pour les policières, sachant notamment que plusieurs chefs d'équipe au pays considèrent que la famille ne doit pas empiéter sur le travail (Duxbury et Higgins 2012)? En même temps, comment tenir compte de la persistance d'une prise en charge plus grande des tâches liées à la sphère domestique par les femmes ${ }^{13}$, tout en évitant de faire porter principalement aux policières le poids des revendications en matière de conciliation travail-famille? De telles revendications peuvent venir ébranler certains aspects de la culture organisationnelle policière. Et, comme le souligne Beauchesne (2007), l'idéologie professionnelle, se traduisant dans la culture organisationnelle, constitue un nœud de résistance. Les policières connaissent bien ce «nœud » qui, quand on tente de le desserrer, peut générer un ressac. Sans oublier que les mesures spéciales d'accommodement qui s'adressent aux policières ont, selon Beauchesne (2007), peu d'impact sur la culture organisationnelle et peuvent entraîner des perceptions de traitement de faveur à leur égard (Kurtz 2012).

En d'autres termes, les stratégies transformatrices doivent clairement s'articuler en matière de changements dans la culture organisationnelle et être officialisées à la fois par des mesures et par l'appui au sein de l'organisation policière (autant par l'employeur que par les collègues).

\section{Conclusion}

Sachant qu'il y a quelques années, le commissaire de la GRC (2012:6) a rehaussé l'« objectif-repère » des effectifs féminin à $35 \%$, nous pouvons aisément présumer que la réflexion et les actions favorisant la conciliation travail-famille seront de plus en plus présentes dans le paysage organisationnel. La littérature à propos de la conciliation travail-famille au sein de l'organisation policière au Canada (Agocs, Langan et Sanders 2015; GRC 2012 et 2013; Duxbury et Higgins 2012; Tremblay, Génin et Di Loreto 2011) amène à croire que les corps policiers qui tardent à se pencher sur la question risquent d'en vivre les répercussions, peinant à recruter et à maintenir en poste les policières, mais également les policiers, qui opteront pour des employeurs qui leur offriront des conditions de travail et une culture professionnelle favorisant l'équilibre entre vie personnelle et vie professionnelle (Duxbury et Higgins 2012; GRC 2012 et 2013) ${ }^{14}$.

13 Dans leur recherche, Agocs, Langan et Sanders (2015:282) montrent « the persistence of gender inequality in the domestic realm, even though the police mothers are working in a male-dominated profession, often with husbands who are also police officers. This finding runs contrary to a widely held belief that gender inequalities are a " thing of the past", a dominant ideology that persists especially when men and women are in the same occupation. »

14 Une littérature de plus en plus étoffée montre que le niveau de satisfaction au travail, le niveau de stress vécu au quotidien, la santé mentale, le sentiment d'avoir un certain contrôle sur les sphères personnelle et professionnelle, le niveau d'absentéisme au travail et le maintien en emploi sont différentes facettes d'un même défi, soit la conciliation travail- 
Nous avons présenté plus haut quelques données sur l'état de la conciliation travail-famille dans les conventions collectives des organisations policières canadiennes. Soulignons particulièrement quatre points :

- premièrement, bien que plusieurs types d'horaire de travail semblent exister au sein des organisations policières canadiennes, les conventions collectives ne nous renseignent pas sur la facilité d'accès à ces horaires;

- deuxièmement, le temps partiel ne constitue pas une mesure privilégiée par les grands corps policiers canadiens; le partage de poste semble une avenue davantage développée;

- troisièmement, le quart des organisations policières à l'étude limite les prestations supplémentaires de chômage au congé de maternité, et n'offrent pas le même supplément pour le congé parental (défavorisant notamment les policiers);

- quatrièmement, les congés pour obligation familiale constituent une mesure peu développée. Lorsqu'ils sont possibles, ces congés sont souvent peu généreux en termes de durée ou de rémunération.

Il va sans dire que d'autres recherches seront nécessaires pour tracer un portrait plus complet de la conciliation travail-famille au sein de l'organisation policière canadienne. Des recherches portant plus particulièrement sur les pratiques qui règnent dans les différents corps policiers, ainsi que sur les perceptions des policières et des policiers quant à la prise en considération de leur situation familiale par l'organisation policière sont autant de pistes de réflexion à poursuivre.

\section{RÉFÉRENCES}

AGOCS, Tricia, Debra LANGAN et Carrie B. SANDERS

2015 «Police Mothers at Home », Gender \& Society, 29, 2 : 265-289.

ASSOCIATION CANADIENNE POUR LA SANTÉ MENTALE, DIVISION DE L'ONTARIO

2015 «Work-Life Balance », Workplace Mental Health Promotion - A How-To Guide, [En ligne], [wmhp.cmhaontario.ca/workplace-mental-health-coreconcepts-issues/issues-in-the-workplace-that-affect-employee-mental-health /work-life-balance] (9 septembre 2016).

\section{ASSOCIATION DES POLICIĖRES ET POLICIERS PROVINCIAUX DU} QUÉBEC

2010 Contrat de travail 2010-2015. Sainte-Julie, Association des policières et policiers provinciaux du Québec.

famille (Agocs, Langan et Sanders 2015; Duxbury et Higgins 2012; Fagan et autres 2012; Fusulier, Sanchez et Ballatore 2013; St-Amour et autres 2005; Statistique Canada 2008; Tremblay et Di Loreto et Génin 2009). 
LA CONCILIATION TRAVAIL-FAMILLE : L'ORGANISATION POLICIÈRE $\mid 227$

BEAUCHESNE, Line

2007 Etre policière: une profession masculine. Montréal, Éditions Cursus universitaire.

BUSSON, Beverly

1997 «Transformer les comportements traditionnels et les attitudes à l'égard des policières », Les femmes dans la police au Canada : les années 2000 et après - Les défis : actes du séminaire, 20-23 mai, Ottawa, Collège canadien de police.

CALGARY POLICE ASSOCIATION

2012 2011-2014 Calgary Police Association Agreement. Calgary, Calgary Police Association.

CHARLESWORTH, Sara, Mark KEEN et Kerri WHITTENBURY

2009 «Integrating Part-time Work in Policing Services: Policy, Practice, and Potential », Police Practice and Research, 10, 1 : 31-47.

DIOTTE, Michèle

2001 Les perceptions des policières dont le conjoint est policier : carrières, vie de couple, famille; étude exploratoire. Thèse de maîtrise. Ottawa, Université d'Ottawa.

DUXBURY, Linda-Elisabeth, et Christophe HIGGINS

2012 Caring for and about Those Who Serve Work-life Conflict and Employee Well Being within Canada's Police. Ottawa, Carleton University.

EDMONTON POLICE ASSOCIATION

2013 Collective Agreement July 14, 2013 to December 29, 2013. Edmonton, Edmonton Police Association.

FAGAN, Colette, et autres

2012 «The Influence of Working Time Arrangements on Work-life Integration or "Balance": A Review of the International Evidence ", IDEAS Working Paper Series from RePEc, [En ligne], [www.ilo.org/wcmsp5/groups/public/ @ed_protect/@protrav/@travail/documents/publication/wcms_187306.pdf] (10 février 2016).

FRATERNITÉ DES POLICIERS ET POLICIÈRES DE MONTRÉAL

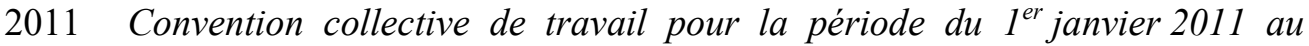
31 décembre 2014. Montréal, Fraternité des policiers et policières de Montréal.

FUSULIER, Bernard, Émilie SANCHEZ et Magali BALLATORE

2013 «Appartenance professionnelle et articulation travail/famille. Comparaison de deux groupes professionnels : les infirmières et les policiers », Enfances, Familles, Générations, 18 : 94-119. 
DIOTTE | 228

GAUTHIER, Madeleine

2008 «Insertion professionnelle des policiers des générations $\mathrm{X}$ et $\mathrm{Y}$ : bilan raisonné de la littérature », Institut national de la recherche scientifique Urbanisation, Culture et Société, [En ligne], [www.enpq.qc.ca/fileadmin/Fi chiers_client/centre_documentaire/InsProfessionnelle.pdf] (10 février 2016).

GENDARMERIE ROYALE DU CANADA (GRC)

2013 «Égalité entre les sexes et respect. Plan d'action de la GRC», Gendarmerie royale du Canada, [En ligne], [www.rcmp-grc.gc.ca/gba-eces/action/indexfra.htm] (10 février 2016).

2012 «Examen comparatif entre les sexes », Gendarmerie royale du Canada, [En ligne], [www.rcmp-grc.gc.ca/aud-ver/reports-rapports/gba-eces/gba-ecesfra.pdf] (10 février 2016).

GOUVERNEMENT DU MANITOBA

2013 «Les femmes et la police au Canada : dossier d'information et document de discussion », Gouvernement du Manitoba, [En ligne], [www.gov.mb.ca/msw/ publications/women_in_policing.fr.pdf] (10 février 2016).

GREENHAUS, Jeffrey H., et Nicholas J. BEUTELL

1985 "Sources of Conflict between Work and Family Roles », The Academy of Management Review, 10, $1: 76-88$.

HÉBERT, Gérard, et autres

2007 La convention collective au Québec. Montréal, GMorin.

KURTZ, Don. L.

2012 «Roll Call and the Second Shift: The Influences of Gender and Family on Police Stress », Police Practice and Research, 13, 1: 71-86, [En ligne], [doi.org/10.1080/15614263.2011.596714] (9 mai 2016).

MONTGOMERY, Ruth

2012 «L'établissement de bilans de l'égalité entre les sexes dans les organisations policières », Condition féminine Canada, [En ligne], [www.swc-cfc.gc.ca/rccr/gapo-ebop/gapo-ebop-fra.pdf] (11 février 2016).

ONTARIO PROVINCIAL POLICE

2011 « Destination Diversity. The Ontario Provincial Police Diversity Journey », Public Safety Canada, [En ligne], [www.publicsafety.gc.ca/lbrr/archives/cn mcs-plcng/cn31049-eng.pdf] (9 mai 2016).

ONTARIO PROVINCIAL POLICE ASSOCIATION

2008 OPPA Uniform Collective Agreement 2009-2011. Barrie, Ontario Provincial Police Association.

POLICE PROVINCIALE DE L'ONTARIO

2015 «Rapport annuel 2014 de la Police provinciale de l'Ontario», Police provinciale de l'Ontario, [En ligne], [www.opp.ca/index.php?\&lng=fr\&id= 115\&entryid=56c22aac8f94acff6aa009d0] (10 février 2016). 
LA CONCILIATION TRAVAIL-FAMILLE : L'ORGANISATION POLICIÈRE $\mid 229$

ROYAL NEWFOUNDLAND CONSTABULARY

2011 «Safer Communities through Policing Excellence. Corporate Plan 2011$2014 »$, Royal Newfoundland Constabulary, [En ligne], [www.rnc.gov.nl.ca/ publications/pdf/2011_2014_corporate_plan.pdf] (10 février 2016).

SCHULZE, Corina

2011 «Family Leave and Law Enforcement : A Survey of Parents in U.S. Police Departments », Critical Criminology, 19, 2 : 137-153, [En ligne], [doi.org/1 0.1007/s10612-010-9108-6] (9 mars 2016).

SERVICE DE POLICE DE LA VILLE DE MONTRÉAL

2006 «Une police à l'image de sa population. Programme d'accès à l'égalité pour le personnel policier », Service de police de la Ville de Montréal, [En ligne], [www.spvm.qc.ca/upload/documentations/Bilan_PAE_2006.pdf] (12 septembre 2016).

ST-AMOUR, Nathalie, et autres

2005 La difficulté de concilier travail-famille : ses impacts sur la santé physique et mentale des familles québécoises. Rapport de la Direction développement des individus et des communautés. Québec, Institut national de santé publique du Québec.

STATISTIQUE CANADA

2015 «Les ressources policières au Canada, 2014 », Juristat, [En ligne], [www.statcan.gc.ca/pub/85-002-x/2015001/article/14146-fra.pdf]

(11 septembre 2016).

2014 «Les ressources policières au Canada, 2013 », Juristat, [En ligne], [www.statcan.gc.ca/pub/85-002-x/2014001/article/11914-fra.pdf] (2 février 2016).

2008 «Work-life Balance of Shift Workers », Perspectives on Labour and Income - Statistics Canada, 9, 8 : 5-16.

SÛRETÉ DU QUÉBEC

2014 «Rapport annuel de gestion 2013-2014», Sûreté du Québec, [En ligne], [www.sq.gouv.qc.ca/mission-et-services/publications/rapport-annuel-degestion-2013-2014.pdf] (10 février 2016).

TEAMSTER LOCAL N.31

2012 Collective Agreement Vancouver Police 2012-2015. Delta (BC), Teamsters Union Local 31.

TORONTO POLICE ASSOCIATION

2011 Collective Agreement 2011-2014. Toronto, Toronto Police Association.

TREMBLAY, Diane-Gabrielle

2012 Articuler emploi et famille : le rôle du soutien organisationnel au cœur de trois professions. Québec, Presses de l'Université du Québec.

TREMBLAY, Diane-Gabrielle, et Émilie GÉNIN

2011 «Parental Leave: An Important Employee Right, But an Organizational Challenge », Employ Respons Rights Journal, 23 : 249-268. 
DIOTTE $\mid 230$

TREMBLAY, Diane-Gabrielle, Martine DI LORETO et Émilie GÉNIN

2009 «Le soutien organisationnel à la conciliation emploi-famille: quelques observations dans le secteur policier au Québec », Note de recherche $n^{\circ} 2009$ 5 de l'Alliance de recherche université-communauté sur la gestion des âges et des temps sociaux, [En ligne], [benhur.teluq.uquebec.ca/SPIP/aruc/IMG/ pdf_ARUC-NR09-05-2.pdf] (5 mars 2016).

TREMBLAY, Diane-Gabrielle, Émilie GÉNIN et Martine DI LORETO

2011 «Advances and Ambivalences : Organisational Support to Work-life Balance in a Police Service », Employment Relations Record, 11, 2 : 75-93.

TREMBLAY, Diane-Gabrielle, et Nadia LAZZARI DODELER

2000 Les pères et la prise du congé parental ou de paternité : une nouvelle réalité. Québec, Presses de l'Université du Québec.

WINNIPEG POLICE ASSOCIATION

2014 Collective Agreement 2012-2016. Winnipeg, Winnipeg Police Association. 